\title{
Competitive Analysis for Two Variants of Online Metric Matching Problem
}

\section{AUTHOR(S):}

Itoh, Toshiya; Miyazaki, Shuichi; Satake, Makoto

\section{CITATION:}

Itoh, Toshiya ...[et al]. Competitive Analysis for Two Variants of Online Metric Matching Problem. Combinatorial Optimization and Applications 2020: 486-498

\section{ISSUE DATE:}

2020

URL:

http://hdl.handle.net/2433/261774

\section{RIGHT:}

This is a post-peer-review, pre-copyedit version of an article published in Combinatorial Optimization and Applications. The final authenticated version is available online at: http://dx.doi.org/10.1007/978-3-030-64843-5 33.; The full-text file will be made open to the public on 4 December 2021 in accordance with publisher's 'Terms and Conditions for SelfArchiving.'; This is not the published version. Please cite only the published version.; この論文は出版社版でありません

。引用の際には出版社版をご確認ご利用ください。 


\title{
Competitive Analysis for Two Variants of Online Metric Matching Problem ${ }^{\star}$
}

\author{
Toshiya Itoh ${ }^{1[0000-0002-1149-7046]}$, Shuichi Miyazaki ${ }^{20000-0003-0369-1970]}$, and \\ Makoto Satake ${ }^{3}$ \\ 1 Department of Mathematical and Computing Science, Tokyo Institute of \\ Technology, 2-12-1 Ookayama, Meguro-ku, Tokyo 152-8550, Japan \\ titoh@c.titech.ac.jp \\ 2 Academic Center for Computing and Media Studies, Kyoto University, \\ Yoshida-Honmachi, Sakyo-ku, Kyoto 606-8501, Japan \\ shuichi@media.kyoto-u.ac.jp \\ 3 Graduate School of Informatics, Kyoto University, \\ Yoshida-Honmachi, Sakyo-ku, Kyoto 606-8501, Japan \\ satake@net.ist.i.kyoto-u.ac.jp
}

\begin{abstract}
In this paper, we study two variants of the online metric matching problem. The first problem is the online metric matching problem where all the servers are placed at one of two positions in the metric space. We show that a simple greedy algorithm achieves the competitive ratio of 3 and give a matching lower bound. The second problem is the online facility assignment problem on a line, where servers have capacities, servers and requests are placed on 1-dimensional line, and the distances between any two consecutive servers are the same. We show lower bounds $1+\sqrt{6}(>3.44948), \frac{4+\sqrt{73}}{3}(>4.18133)$ and $\frac{13}{3}(>4.33333)$ on the competitive ratio when the numbers of servers are 3,4 and 5 , respectively.
\end{abstract}

Keywords: Online algorithm, Competitive analysis, Online matching problem

\section{Introduction}

The online metric matching problem was introduced independently by Kalyanasundaram and Pruhs [7] and Khuller, Mitchell and Vazirani [10]. In this problem, $n$ servers are placed on a given metric space. Then $n$ requests, which are points on the metric space, are given to the algorithm one-by-one in an online fashion. The task of an online algorithm is to match each request immediately to one of $n$ servers. If a request is matched to a server, then it incurs a cost which is equivalent to the distance between them. The goal of the problem is to minimize the sum of the costs. The papers [7] and [10] presented a deterministic online

\footnotetext{
* This work was partially supported by the joint project of Kyoto University and Toyota Motor Corporation, titled "Advanced Mathematical Science for Mobility Society" and JSPS KAKENHI Grant Numbers JP16K00017 and JP20K11677.
} 
algorithm (called Permutation in [7]) and showed that it is (2n-1)-competitive and optimal.

In 1998, Kalyanasundaram and Pruhs [8] posed a question whether we can have a better competitive ratio by restricting the metric space to a line, and introduced the problem called the online matching problem on a line. Since then, this problem has been extensively studied, but there still remains a large gap between the best known lower bound 9.001 [5] and upper bound $O(\log n)$ [16] on the competitive ratio.

In 2020, Ahmed, Rahman and Kobourov [1] proposed a problem called the online facility assignment problem and considered it on a line, which we denote $O F A L$ for short. In this problem, all the servers (which they call facilities) and requests (which they call customers) lie on a 1-dimensional line, and the distance between every pair of adjacent servers is the same. Also, each server has a capacity, which is the number of requests that can be matched to the server. In their model, all the servers are assumed to have the same capacity. Let us denote $\operatorname{OFAL}(k)$ the OFAL problem where the number of servers is $k$. Ahmed et al. [1] showed that for $\operatorname{OFAL}(k)$ the greedy algorithm is $4 k$-competitive for any $k$ and a deterministic algorithm Optimal-fill is $k$-competitive for any $k>2$.

\subsection{Our contributions}

In this paper, we study a variant of the online metric matching problem where all the servers are placed at one of two positions in the metric space. This is equivalent to the case where there are two servers with capacities. We show that a simple greedy algorithm achieves the competitive ratio of 3 for this problem, and show that any deterministic online algorithm has competitive ratio at least 3 .

We also study $\operatorname{OFAL}(k)$ for small $k$. Specifically, we show lower bounds $1+\sqrt{6}$ $(>3.44948), \frac{4+\sqrt{73}}{3}(>4.18133)$ and $\frac{13}{3}(>4.33333)$ on the competitive ratio for OFAL(3), OFAL(4) and $\operatorname{OFAL}(5)$, respectively. We remark that our lower bounds $1+\sqrt{6}$ for $\operatorname{OFAL}(3)$ and $\frac{4+\sqrt{73}}{3}$ for $\operatorname{OFAL}(4)$ do not contradict the abovementioned upper bound of Optimal-fill, since upper bounds by Ahmed et al. [1] are with respect to the asymptotic competitive ratio, while our lower bounds are with respect to the strict competitive ratio (see Sec. 2.3).

\subsection{Related work}

In 1990, Karp, Vazirani and Vazirani [9] first studied an online version of the matching problem. They studied the online matching problem on unweighted bipartite graphs with $2 n$ vertices that contain a perfect matching, where the goal is to maximize the size of the obtained matching. In [9], they first showed that a deterministic greedy algorithm is $\frac{1}{2}$-competitive and optimal. They also presented a randomized algorithm Ranking and showed that it is $\left(1-\frac{1}{e}\right)$-competitive and optimal. See [12] for a survey of the online matching problem. 
As mentioned before, Kalyanasundaram and Pruhs [7] studied the online metric matching problem and showed that the algorithm Permutation is $(2 n-1)$ competitive and optimal. Probabilistic algorithms for this problem were studied in $[4,13]$.

Kalyanasundaram and Pruhs [8] studied the online matching problem on a line. They gave two conjectures that the competitive ratio of this problem is 9 and that the Work-Function algorithm has a constant competitive ratio, both of which were later disproved in [11] and [5], respectively. This problem was studied in $[2,3,6,14-16]$, and the best known deterministic algorithm is the Robust Matching algorithm [15], which is $\Theta(\log n)$-competitive [14,16].

Besides the problem on a line, Ahmed, Rahman and Kobourov [1] studied the online facility assignment problem on an unweighted graph $G(V, E)$. They showed that the greedy algorithm is $2|E|$-competitive and Optimal-Fill is $\frac{|E| k}{r}$ competitive, where $|E|$ is the number of edge of $G$ and $r$ is the radius of $G$.

\section{Preliminaries}

In this section, we give definitions and notations.

\subsection{Online metric matching problem with two servers}

We define the online metric matching problem with two servers, denoted $\operatorname{OMM}(2)$ for short. Let $(X, d)$ be a metric space, where $X$ is a (possibly infinite) set of points and $d(\cdot, \cdot)$ is a distance function. Let $S=\left\{s_{1}, s_{2}\right\}$ be a set of servers and $R=\left\{r_{1}, r_{2}, \ldots, r_{n}\right\}$ be a set of requests. A server $s_{i}$ is characterized by the position $p\left(s_{i}\right) \in X$ and the capacity $c_{i}$ that satisfies $c_{1}+c_{2}=n$. This means that $s_{i}$ can be matched with at most $c_{i}$ requests $(i=1,2)$. A request $r_{i}$ is also characterized by the position $p\left(r_{i}\right) \in X$.

$S$ is given to an online algorithm in advance, while requests are given oneby-one from $r_{1}$ to $r_{n}$. At any time of the execution of an algorithm, a server is called free if the number of requests matched with it is less than its capacity, and full otherwise. When a request $r_{i}$ is revealed, an online algorithm must match $r_{i}$ with one of free servers. If $r_{i}$ is matched with the server $s_{j}$, the pair $\left(r_{i}, s_{j}\right)$ is added to the current matching and the cost $d\left(r_{i}, s_{j}\right)$ is incurred for this pair. The cost of the matching is the sum of the costs of all the pairs contained in it. The goal of $\operatorname{OMM}(2)$ is to minimize the cost of the final matching.

\subsection{Online facility assignment problem on a line}

We give the definition of the online facility assignment problem on a line with $k$ servers, denoted $\operatorname{OFAL}(k)$. We state only differences from Sec. 2.1. The set of servers is $S=\left\{s_{1}, s_{2}, \ldots, s_{k}\right\}$ and all the servers have the same capacity $\ell$, i.e., $c_{i}=\ell$ for all $i$. The number of requests must satisfy $n \leq \sum_{i=1}^{k} c_{i}=k \ell$. All the servers and requests are placed on a real number line, so their positions are expressed by a real, i.e., $p\left(s_{i}\right) \in \mathbb{R}$ and $p\left(r_{j}\right) \in \mathbb{R}$. Accordingly, the distance 
function is written as $d\left(r_{i}, s_{j}\right)=\left|p\left(r_{i}\right)-p\left(s_{j}\right)\right|$. We assume that the servers are placed in an increasing order of their indices, i.e., $p\left(s_{1}\right) \leq p\left(s_{2}\right) \leq \ldots \leq p\left(s_{k}\right)$. In this problem, any distance between two consecutive servers is the same, that is, $\left|p\left(s_{i}\right)-p\left(s_{i+1}\right)\right|=d(1 \leq i \leq k-1)$ for some constant $d$. Without loss of generality, we let $d=1$.

\subsection{Competitive ratio}

To evaluate the performance of an online algorithm, we use the strict competitive ratio. (Hereafter, we omit "strict".) For an input $\sigma$, let $A L G(\sigma)$ and $O P T(\sigma)$ be the costs of the matchings obtained by an online algorithm $A L G$ and an optimal offline algorithm $O P T$, respectively. Then the competitive ratio of $A L G$ is the supremum of $c$ that satisfies $A L G(\sigma) \leq c \cdot O P T(\sigma)$ for any input $\sigma$.

\section{Online Metric Matching Problem with Two Servers}

\section{$3.1 \quad$ Upper bound}

In this section, we define a greedy algorithm GREEDY for $\operatorname{OMM}(2)$ and show that it is 3-competitive.

Definition 1. When a request is given, GREEDY matches it with the closest free server. If a given request is equidistant from the two servers and both servers are free, GREEDY matches this request with $s_{1}$.

In the following discussion, we fix an optimal offline algorithm $O P T$. If a request $r$ is matched with the server $s_{x}$ by GREEDY and with $s_{y}$ by $O P T$, we say that $r$ is of type $\left\langle s_{x}, s_{y}\right\rangle$. We then define some properties of inputs.

Definition 2. Let $\sigma$ be an input to $O M M(2)$. If every request in $\sigma$ is matched with a different server by GREEDY and OPT, $\sigma$ is called anti-opt.

Definition 3. Let $\sigma$ be an input to OMM(2). Suppose that GREEDY matches its first request $r_{1}$ to the server $s_{x} \in\left\{s_{1}, s_{2}\right\}$. If GREEDY matches $r_{1}$ through $r_{c_{x}}$ to $s_{x}$ (note that $c_{x}$ is the capacity of $s_{x}$ ) and $r_{c_{x}+1}$ through $r_{n}$ to the other server $s_{3-x}, \sigma$ is called one-sided-priority.

By the following two lemmas, we show that it suffices to consider inputs that are anti-opt and one-sided-priority. For an input $\sigma$, we define $\operatorname{Rate}(\sigma)$ as

$$
\operatorname{Rate}(\sigma)= \begin{cases}\frac{G R E E D Y(\sigma)}{O P T(\sigma)} & (\text { if } \operatorname{OPT}(\sigma) \neq 0) \\ 1 & \text { (if } \operatorname{OPT}(\sigma)=\operatorname{GREEDY}(\sigma)=0) \\ \infty & \text { (if } \operatorname{OPT}(\sigma)=0 \text { and } \operatorname{GREEDY}(\sigma)>0)\end{cases}
$$

Lemma 1. For any input $\sigma$, there exists an anti-opt input $\sigma^{\prime}$ such that $\operatorname{Rate}\left(\sigma^{\prime}\right) \geq \operatorname{Rate}(\sigma)$. 
Proof. If $\sigma$ is already anti-opt, we can set $\sigma^{\prime}=\sigma$. Hence, in the following, we assume that $\sigma$ is not anti-opt. Then there exists a request $r$ in $\sigma$ that is matched with the same server $s_{x}$ by $O P T$ and GREEDY. Let $\sigma^{\prime \prime}$ be an input obtained from $\sigma$ by removing $r$ and subtracting the capacity of $s_{x}$ by 1 . By this modification, neither OPT nor GREEDY changes a matching for the remaining requests. Therefore, GREEDY $\left(\sigma^{\prime \prime}\right)=\operatorname{GREEDY}(\sigma)-d\left(r, s_{x}\right)$ and $O P T\left(\sigma^{\prime \prime}\right)=$ $O P T(\sigma)-d\left(r, s_{x}\right)$, which implies Rate $\left(\sigma^{\prime \prime}\right) \geq \operatorname{Rate}(\sigma)$.

Let $\sigma^{\prime}$ be the input obtained by repeating this operation until the input sequence becomes anti-opt. Then $\sigma^{\prime}$ satisfies the conditions of this lemma.

Lemma 2. For any anti-opt input $\sigma$, there exists an anti-opt and one-sidedpriority input $\sigma^{\prime}$ such that Rate $\left(\sigma^{\prime}\right)=\operatorname{Rate}(\sigma)$.

Proof. If $\sigma$ is already one-sided-priority, we can set $\sigma^{\prime}=\sigma$. Hence, in the following, we assume that $\sigma$ is not one-sided-priority.

Since $\sigma$ is anti-opt, $\sigma$ contains only requests of type $\left\langle s_{1}, s_{2}\right\rangle$ or $\left\langle s_{2}, s_{1}\right\rangle$. Without loss of generality, assume that in execution of GREEDY, the server $s_{1}$ becomes full before $s_{2}$, and let $r_{t}$ be the request that makes $s_{1}$ full (i.e., $r_{t}$ is the last request of type $\left\langle s_{1}, s_{2}\right\rangle$ ).

Because $\sigma$ is not one-sided-priority, $\sigma$ includes at least one request $r_{i}$ of type $\left\langle s_{2}, s_{1}\right\rangle$ before $r_{t}$. Let $\sigma^{\prime \prime}$ be the input obtained from $\sigma$ by moving $r_{i}$ to just after $r_{t}$. Since the set of requests is unchanged in $\sigma$ and $\sigma^{\prime \prime}$, an optimal matching for $\sigma$ is also optimal for $\sigma^{\prime \prime}$, so $O P T\left(\sigma^{\prime \prime}\right)=O P T(\sigma)$. In the following, we show that GREEDY matches each request to the same server in $\sigma$ and $\sigma^{\prime \prime}$. The sequence of requests up to $r_{i-1}$ are the same in $\sigma^{\prime \prime}$ and $\sigma$, so the claim clearly holds for $r_{1}$ through $r_{i-1}$. The behavior of GREEDY for $r_{i+1}$ through $r_{t}$ in $\sigma^{\prime \prime}$ is also the same for those in $\sigma$, because when serving these requests, both $s_{1}$ and $s_{2}$ are free in both $\sigma$ and $\sigma^{\prime \prime}$. Just after serving $r_{t}$ in $\sigma^{\prime \prime}, s_{1}$ becomes full, so GREEDY matches $r_{i}, r_{t+1}, \ldots, r_{n}$ with $s_{2}$ in $\sigma^{\prime \prime}$. Note that these requests are also matched with $s_{2}$ in $\sigma$. Hence GREEDY $\left(\sigma^{\prime \prime}\right)=\operatorname{GREEDY}(\sigma)$ and it results that $\operatorname{Rate}\left(\sigma^{\prime \prime}\right)=\operatorname{Rate}(\sigma)$. Note that $\sigma^{\prime \prime}$ remains anti-opt.

Let $\sigma^{\prime}$ be the input obtained by repeating this operation until the input sequence becomes one-sided-priority. Then $\sigma^{\prime}$ satisfies the conditions of this lemma.

We can now prove the upper bound.

Theorem 1. The competitive ratio of GREEDY is at most 3 for $O M M(2)$.

Proof. By Lemma 1, it suffices to analyze only anti-opt inputs. In an anti-opt input, the number of requests of type $\left\langle s_{1}, s_{2}\right\rangle$ and that of type $\left\langle s_{2}, s_{1}\right\rangle$ are the same and the capacities of $s_{1}$ and $s_{2}$ are $n / 2$ each. By Lemma 2, it suffices to analyze only the inputs where the first $n / 2$ requests are of type $\left\langle s_{1}, s_{2}\right\rangle$ and the remaining $n / 2$ requests are of type $\left\langle s_{2}, s_{1}\right\rangle$.

Let $\sigma$ be an arbitrary such input. Then we have that

$$
\operatorname{GREEDY}(\sigma)=\sum_{i=1}^{n / 2} d\left(r_{i}, s_{1}\right)+\sum_{i=n / 2+1}^{n} d\left(r_{i}, s_{2}\right)
$$


and

$$
O P T(\sigma)=\sum_{i=1}^{n / 2} d\left(r_{i}, s_{2}\right)+\sum_{i=n / 2+1}^{n} d\left(r_{i}, s_{1}\right) .
$$

When serving $r_{1}, r_{2}, \ldots, r_{n / 2}$, both servers are free but GREEDY matched them with $s_{1}$. Hence $d\left(r_{i}, s_{1}\right) \leq d\left(r_{i}, s_{2}\right)$ for $1 \leq i \leq n / 2$. By the triangle inequality, we have $d\left(r_{i}, s_{2}\right) \leq d\left(s_{1}, s_{2}\right)+d\left(r_{i}, s_{1}\right)$ for $n / 2+1 \leq i \leq n$. Again, by the triangle inequality, we have $d\left(s_{1}, s_{2}\right) \leq d\left(r_{i}, s_{1}\right)+d\left(r_{i}, s_{2}\right)$ for $1 \leq i \leq n$.

From these inequalities, we have that

$$
\begin{aligned}
\operatorname{GREEDY}(\sigma) & =\sum_{i=1}^{n / 2} d\left(r_{i}, s_{1}\right)+\sum_{i=n / 2+1}^{n} d\left(r_{i}, s_{2}\right) \\
& \leq \sum_{i=1}^{n / 2} d\left(r_{i}, s_{2}\right)+\sum_{i=n / 2+1}^{n}\left(d\left(s_{1}, s_{2}\right)+d\left(r_{i}, s_{1}\right)\right) \\
& =O P T(\sigma)+\frac{n}{2} d\left(s_{1}, s_{2}\right) \\
& =O P T(\sigma)+\frac{1}{2} \sum_{i=1}^{n} d\left(s_{1}, s_{2}\right) \\
& \leq O P T(\sigma)+\frac{1}{2} \sum_{i=1}^{n}\left(d\left(r_{i}, s_{1}\right)+d\left(r_{i}, s_{2}\right)\right) \\
& =O P T(\sigma)+\frac{1}{2}(O P T(\sigma)+G R E E D Y(\sigma)) \\
& =\frac{3}{2} O P T(\sigma)+\frac{1}{2} G R E E D Y(\sigma) .
\end{aligned}
$$

Thus GREEDY $(\sigma) \leq 3 O P T(\sigma)$ and the competitive ratio of GREEDY is at most 3 .

\section{$3.2 \quad$ Lower bound}

Theorem 2. The competitive ratio of any deterministic online algorithm for $O M M(2)$ is at least 3.

Proof. We prove this lower bound on a 1-dimensional real line metric. Let $p\left(s_{1}\right)=$ $-d$ and $p\left(s_{2}\right)=d$ for a constant $d$. Consider any deterministic algorithm $A L G$. First, our adversary gives $c_{1}-1$ requests at $p\left(s_{1}\right)$ and $c_{2}-1$ requests at $p\left(s_{2}\right)$. $O P T$ matches the first $c_{1}-1$ requests with $s_{1}$ and the rest with $s_{2}$. If there exists a request that $A L G$ matches differently from $O P T$, the adversary gives two more requests, one at $p\left(s_{1}\right)$ and the other at $p\left(s_{2}\right)$. Then, the cost of $O P T$ is zero, while the cost of $A L G$ is positive, so the ratio of them becomes infinity.

Next, suppose that $A L G$ matches all these requests with the same server as $O P T$. Then the adversary gives the next request at the origin 0 . Let $s_{x}$ be the 
server that $A L G$ matches this request with. Then $O P T$ matches this request with the other server $s_{3-x}$. After that, the adversary gives the last request at $p\left(s_{x}\right)$. ALG has to match it with $s_{3-x}$ and $O P T$ matches it with $s_{x}$. The costs of $A L G$ and $O P T$ for this input are $3 d$ and $d$, respectively. This completes the proof.

\section{Online Facility Assignment Problem on Line}

In this section, we show lower bounds on the competitive ratio of $\operatorname{OFAL}(k)$ for $k=3,4$, and 5 . To simplify the proofs, we use Definitions 4 and 5 and Proposition 1 , observed in $[3,11]$, that allow us to restrict online algorithms to consider.

Definition 4. When a request $r$ is given, the surrounding servers for $r$ are the closest free server to the left of $r$ and the closest free server to the right of $r$.

Definition 5. If an algorithm ALG matches every request of an input $\sigma$ with one of the surrounding servers, $A L G$ is called surrounding-oriented for $\sigma$. If $A L G$ is surrounding-oriented for any input, then $A L G$ is called surrounding-oriented.

Proposition 1. For any algorithm ALG, there exists a surrounding-oriented algorithm $A L G^{\prime}$ such that $A L G^{\prime}(\sigma) \leq A L G(\sigma)$ for any input $\sigma$.

The proof of Proposition 1 is omitted in [3,11], so we prove it here for completeness.

Proof. Suppose that $A L G$ is not surrounding-oriented for $\sigma$. Then $A L G$ matches at least one request of $\sigma$ with a non-surrounding server. Let $r$ be the earliest one among such requests and $s$ be the server matched with $r$ by $A L G$. Also let $s^{\prime}$ be the surrounding server (for $r$ ) on the same side as $s$ and $r^{\prime}$ be the request matched with $s^{\prime}$ by $A L G$.

We modify $A L G$ to $A L G^{\prime \prime}$ so that $A L G^{\prime \prime}$ matches $r$ with $s^{\prime}$ and $r^{\prime}$ with $s$ (and behaves the same as $A L G$ for other requests). Without loss of generality, we can assume that $p(r)<p(s)$. Then we have that $p(r) \leq p\left(s^{\prime}\right)<p(s)$. If $p\left(r^{\prime}\right) \leq p\left(s^{\prime}\right)$, then $A L G^{\prime \prime}(\sigma)=A L G(\sigma)$ and if $p\left(r^{\prime}\right)>p\left(s^{\prime}\right)$, then $A L G^{\prime \prime}(\sigma)<A L G(\sigma)$. In either case, we have that $A L G^{\prime \prime}(\sigma) \leq A L G(\sigma)$.

Let $A L G^{\prime \prime \prime}$ be the algorithm obtained by applying this modification as long as there is a request in $\sigma$ matched with a non-surrounding server. Then $A L G^{\prime \prime \prime}(\sigma) \leq$ $A L G(\sigma)$ and $A L G^{\prime \prime \prime}$ is surrounding-oriented for $\sigma$.

We do the above modification for all the inputs for which $A L G$ is not surrounding-oriented, and let $A L G^{\prime}$ be the resulting algorithm. Then $A L G^{\prime}(\sigma) \leq$ $A L G(\sigma)$ and $A L G^{\prime}$ is surrounding-oriented, as required.

By Proposition 1, it suffices to consider only surrounding-oriented algorithms for lower bound arguments.

Theorem 3. The competitive ratio of any deterministic online algorithm for OFAL(3) is at least $1+\sqrt{6}(>3.44948)$. 
Proof. Let $A L G$ be any surrounding-oriented algorithm. Our adversary first gives $\ell-1$ requests at $p\left(s_{i}\right)$ for each $i=1,2$ and 3 . OPT matches every request $r$ with the server at the same position $p(r)$. If $A L G$ matches some request $r$ with a server not at $p(r)$, then the adversary gives three more requests, one at each position of the server. The cost of $A L G$ is positive and the cost of $O P T$ is zero, so the ratio of the costs is infinity.

Next, suppose that $A L G$ matches all these requests to the same server as $O P T$. Let $x=\sqrt{6}-2(\simeq 0.44949)$ and $y=3 \sqrt{6}-7(\simeq 0.34847)$. The adversary gives a request $r_{1}$ at $p\left(s_{2}\right)+x$.

Case 1. $A L G$ matches $r_{1}$ with $s_{3}$.

See Fig. 1. The adversary gives the next request $r_{2}$ at $p\left(s_{3}\right)$. $A L G$ matches it with $s_{2}$. Finally, the adversary gives a request $r_{3}$ at $p\left(s_{1}\right)$ and $A L G$ matches it with $s_{1}$. The cost of $A L G$ is $2-x=4-\sqrt{6}$ and the cost of $O P T$ is $x=\sqrt{6}-2$. The ratio is $\frac{4-\sqrt{6}}{\sqrt{6}-2}=1+\sqrt{6}$.

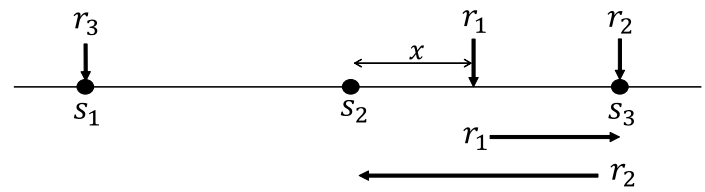

Fig. 1. Requests and $A L G$ 's matching for Case 1 of Theorem 3.

Case 2. ALG matches $r_{1}$ with $s_{2}$.

The adversary gives the next request $r_{2}$ at $p\left(s_{2}\right)-y$. We have two subcases.

Case 2-1. ALG matches $r_{2}$ with $s_{1}$.

See Fig. 2. The adversary gives a request $r_{3}$ at $p\left(s_{1}\right)$ and $A L G$ matches it with $s_{3}$. The cost of $A L G$ is $3+x-y=8-2 \sqrt{6}$ and the cost of $O P T$ is $1-x+y=2 \sqrt{6}-4$. The ratio is $\frac{8-2 \sqrt{6}}{2 \sqrt{6}-4}=1+\sqrt{6}$.

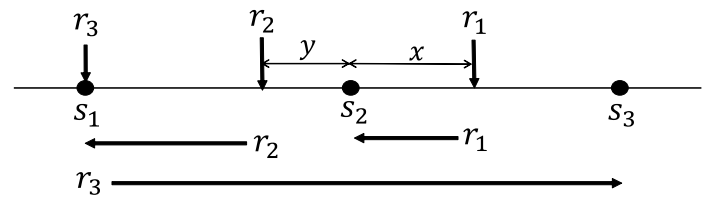

Fig. 2. Requests and $A L G$ 's matching for Case 2-1 of Theorem 3.

Case 2-2. ALG matches $r_{2}$ with $s_{3}$.

See Fig. 3 . The adversary gives a request $r_{3}$ at $p\left(s_{3}\right)$ and $A L G$ matches it with $s_{1}$. 
The cost of $A L G$ is $3+x+y=4 \sqrt{6}-6$ and the cost of $O P T$ is $1+x-y=6-2 \sqrt{6}$. The ratio is $\frac{4 \sqrt{6}-6}{6-2 \sqrt{6}}=1+\sqrt{6}$.

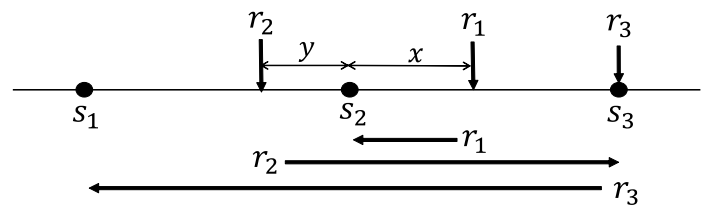

Fig. 3. Requests and $A L G$ 's matching for Case $2-2$ of Theorem 3.

In any case, the ratio of $A L G$ 's cost to $O P T$ 's cost is $1+\sqrt{6}$. This completes the proof.

Theorem 4. The competitive ratio of any deterministic online algorithm for OFAL(4) is at least $\frac{4+\sqrt{73}}{3}(>4.18133)$.

Proof. Let $A L G$ be any surrounding-oriented algorithm. In the same way as the proof of Theorem 3 , the adversary first gives $\ell-1$ requests at $p\left(s_{i}\right)$ for $i=1,2,3$, and 4 , and we can assume that $O P T$ and $A L G$ match each of these requests to the server at the same position. Then, the adversary gives a request $r_{1}$ at $\frac{p\left(s_{2}\right)+p\left(s_{3}\right)}{2}$. Without loss of generality, assume that $A L G$ matches it with $s_{2}$.

Let $x=\frac{10-\sqrt{73}}{2}(\simeq 0.72800)$ and $y=\frac{11 \sqrt{73}-93}{8}(\simeq 0.12301)$. The adversary gives a request $r_{2}$ at $p\left(s_{1}\right)+x$. We consider two cases depending on the behavior of $A L G$.

Case 1. ALG matches $r_{2}$ with $s_{1}$.

See Fig. 4. The adversary gives the next request $r_{3}$ at $p\left(s_{1}\right)$. ALG has to match it with $s_{3}$. Finally, the adversary gives a request $r_{4}$ at $p\left(s_{4}\right)$ and $A L G$ matches it with $s_{4}$. The cost of $A L G$ is $\frac{5}{2}+x=\frac{15-\sqrt{73}}{2}$ and the cost of $O P T$ is $\frac{3}{2}-x=\frac{\sqrt{73}-7}{2}$. The ratio is $\frac{15-\sqrt{73}}{\sqrt{73}-7}=\frac{4+\sqrt{73}}{3}$.

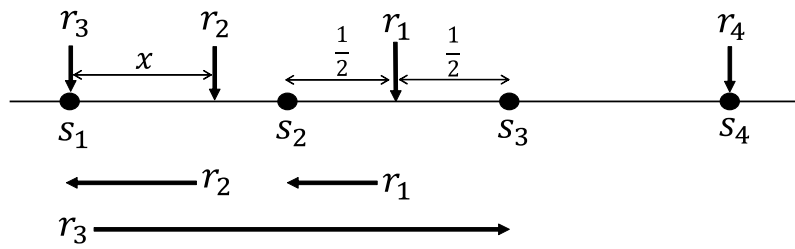

Fig. 4. Requests and $A L G$ 's matching for Case 1 of Theorem 4. 
Case 2. ALG matches $r_{2}$ with $s_{3}$.

The adversary gives the next request $r_{3}$ at $p\left(s_{3}\right)+y$. We have two subcases.

Case 2-1. ALG matches $r_{3}$ with $s_{4}$.

See Fig. 5. The adversary gives a request $r_{4}$ at $p\left(s_{4}\right)$. $A L G$ has to match it with $s_{1}$. The cost of $A L G$ is $\frac{13}{2}-x-y=\frac{105-7 \sqrt{73}}{8}$ and the cost of $O P T$ is $\frac{1}{2}+x+y=\frac{7 \sqrt{73}-49}{8}$. The ratio is $\frac{105-7 \sqrt{73}}{7 \sqrt{73}-49}=\frac{4+\sqrt{73}}{3}$.

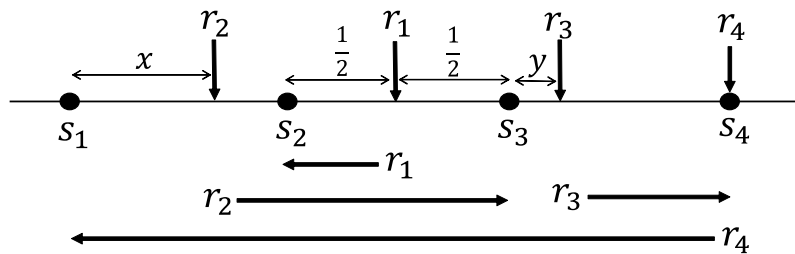

Fig. 5. Requests and $A L G$ 's matching for Case 2-1 of Theorem 4.

\section{Case 2-2. $A L G$ matches $r_{3}$ with $s_{1}$.}

See Fig. 6. The adversary gives a request $r_{4}$ at $p\left(s_{1}\right)$ and $A L G$ has to match it with $s_{4}$. The cost of $A L G$ is $\frac{15}{2}-x+y=\frac{15 \sqrt{73}-73}{8}$ and the cost of $O P T$ is $\frac{5}{2}-x-y=\frac{73-7 \sqrt{73}}{8}$. The ratio is $\frac{15 \sqrt{73}-73}{73-7 \sqrt{73}}=\frac{4+\sqrt{73}}{3}$.

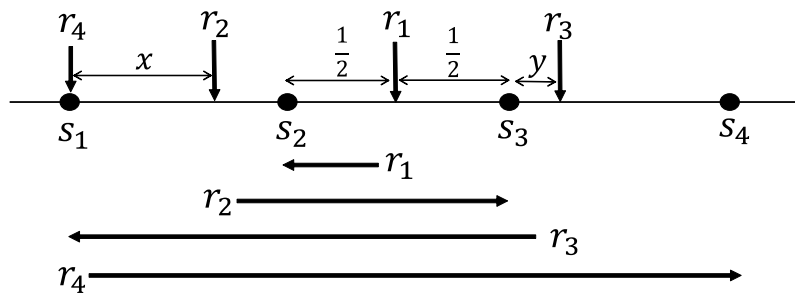

Fig. 6. Requests and $A L G$ 's matching for Case 2-2 of Theorem 4.

In any case, the ratio of $A L G$ 's cost to $O P T$ 's cost is $\frac{4+\sqrt{73}}{3}$. This completes the proof.

Theorem 5. The competitive ratio of any deterministic online algorithms for OFAL(5) is at least $\frac{13}{3}(>4.33333)$.

Proof. Let $A L G$ be any surrounding-oriented algorithm. In the same way as the proof of Theorem 3 , the adversary first gives $\ell-1$ requests at $p\left(s_{i}\right)$ for 
$i=1,2,3,4$, and 5 , and we can assume that $O P T$ and $A L G$ match each of these requests to the server at the same position.

Then, the adversary gives a request $r_{1}$ at $p\left(s_{3}\right)$. If $A L G$ matches this with $s_{2}$ or $s_{4}$, the adversary gives the remaining requests at $p\left(s_{1}\right), p\left(s_{2}\right), p\left(s_{4}\right)$ and $p\left(s_{5}\right)$. OPT's cost is zero, while $A L G$ 's cost is positive, so the ratio is infinity. Therefore, assume that $A L G$ matches $r_{1}$ with $s_{3}$. The adversary then gives a request $r_{2}$ at $p\left(s_{3}\right)$. Without loss of generality, assume that $A L G$ matches it with $s_{2}$. Next, the adversary gives a request $r_{3}$ at $p\left(s_{1}\right)+\frac{7}{8}$. We consider two cases depending on the behavior of $A L G$.

Case 1. $A L G$ matches $r_{3}$ with $s_{1}$.

See Fig. 7. The adversary gives the next request $r_{4}$ at $p\left(s_{1}\right)$. $A L G$ has to match it with $s_{4}$. Finally, the adversary gives a request $r_{5}$ at $p\left(s_{5}\right)$ and $A L G$ matches it with $s_{5}$. The cost of $A L G$ is $\frac{39}{8}$ and the cost of $O P T$ is $\frac{9}{8}$. The ratio is $\frac{13}{3}$.

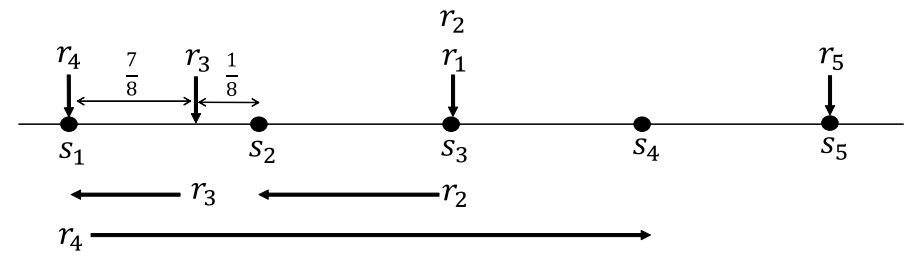

Fig. 7. Requests and $A L G$ 's matching for Case 1 of Theorem 5.

\section{Case 2. $A L G$ matches $r_{3}$ with $s_{4}$.}

The adversary gives the next request $r_{4}$ at $p\left(s_{4}\right)$. We have two subcases.

Case 2-1. ALG matches $r_{4}$ with $s_{1}$.

See Fig. 8. The adversary gives a request $r_{5}$ at $p\left(s_{1}\right)$ and $A L G$ has to match it with $s_{5}$. The cost of $A L G$ is $\frac{81}{8}$ and the cost of $O P T$ is $\frac{17}{8}$. The ratio is $\frac{81}{17}>\frac{13}{3}$.

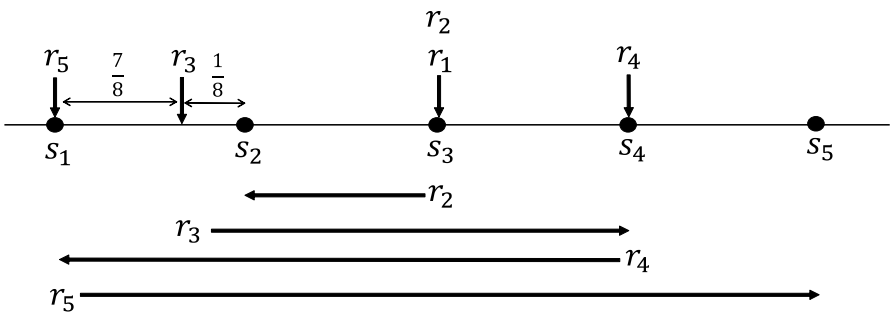

Fig. 8. Requests and $A L G$ 's matching for Case 2-1 of Theorem 5. 
Case 2-2. $A L G$ matches $r_{4}$ with $s_{5}$.

See Fig. 9. The adversary gives a request $r_{5}$ at $p\left(s_{5}\right)$ and $A L G$ has to match it with $s_{1}$. The cost of $A L G$ is $\frac{65}{8}$ and the cost of $O P T$ is $\frac{15}{8}$. The ratio is $\frac{13}{3}$.

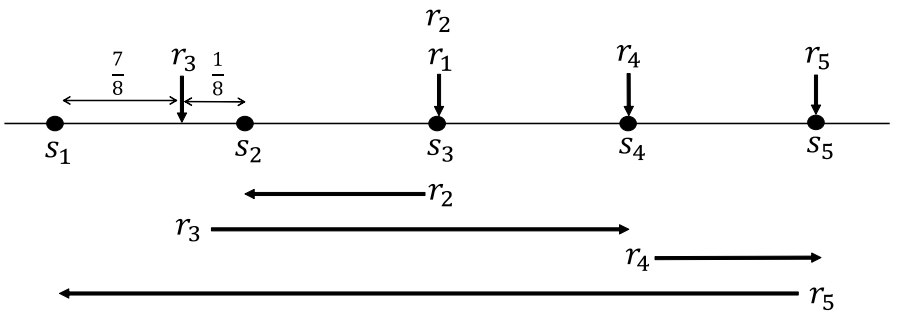

Fig. 9. Requests and $A L G$ 's matching for Case 2-2 of Theorem 5.

In any case, the ratio of $A L G$ 's cost to $O P T$ 's cost is at least $\frac{13}{3}$, which completes the proof.

\section{Conclusion}

In this paper, we studied two variants of the online metric matching problem. The first is a restriction where all the servers are placed at one of two positions in the metric space. For this problem, we presented a greedy algorithm and showed that it is 3-competitive. We also proved that any deterministic online algorithm has competitive ratio at least 3 , giving a matching lower bound. The second variant is the Online Facility Assignment Problem on a line with a small number of servers. We showed lower bounds on the competitive ratio $1+\sqrt{6}$, $\frac{4+\sqrt{73}}{3}$, and $\frac{13}{3}$ when the numbers of servers are 3,4 , and 5 , respectively.

One of the future work is to analyze the online metric matching problem with three or more server positions. Another interesting direction is to consider an optimal online algorithm for the Online Facility Assignment Problem on a line when the numbers of servers are 3,4 , and 5 .

Acknowledgments. The authors would like to thank the anonymous reviewers for their valuable comments.

\section{References}

1. Ahmed, A., Rahman, M., Kobourov, S.: Online facility assignment. Theoretical Computer Science 806, 455-467 (2020)

2. Antoniadis, A., Barcelo, M., Nugent, M., Pruhs, N., Scquizzato, M.: A $o(n)$ competitive deterministic algorithm for online matching on a line. WAOA 2012 pp. 11-22 (2014) 
3. Antoniadis, A., Fischer, C., Tönnis, A.: A collection of lower bounds for online matching on the line. LATIN 2018 pp. 52-65 (2018)

4. Bansal, N., Buchbinder, N., Gupta, A., Naor, J.: An $o\left(\log ^{2} k\right)$-competitive algorithm for metric bipartite matching. ESA 2007 pp. 522-533 (2007)

5. Fuchs, B., Hochstättler, W., Kern, W.: Online matching on a line. Theoretical Computer Science 332, 251-264 (2005)

6. Gupta, A., Lewi, K.: The online metric matching problem for doubling metrics. ICALP 2012 pp. 424-435 (2012)

7. Kalyanasundaram, B., Pruhs, K.: Online weighted matching. Journal of Algorithms 14(3), 478-488 (1993)

8. Kalyanasundaram, B., Pruhs, K.: Online network optimization problems. Online Algorithms. Lecture Notes in Computer Science 1442, 268-280 (1998)

9. Karp, R., Vazirani, U., Vazirani, V.: An optimal algorithm for on-line bipartite matching. STOC 1990 pp. 352-358 (1990)

10. Khuller, S., Mitchell, S., Vazirani, V.: On-line algorithms for weighted bipartite matching and stable marriages. Theoretical Computer Science 127(2), 255-267 (1994)

11. Koutsoupias, E., Nanavati, A.: The online matching problem on a line. WAOA 2003 pp. 179-191 (2004)

12. Mehta, A.: Online matching and ad allocation. Theoretical Computer Science 8(4), 265-368 (2012)

13. Meyerson, A., Nanavati, A., Poplawski, L.: Randomized online algorithms for minimum metric bipartite matching. SODA 2006 pp. 954-959 (2006)

14. Nayyar, K., Raghvendra, S.: An input sensitive online algorithm for the metric bipartite matching problem. FOCS 2017 pp. 505-515 (2017)

15. Raghvendra, S.: A robust and optimal online algorithm for minimum metric bipartite matching. APPROX/RANDOM 2016 60, 18:1-18:16 (2016)

16. Raghvendra, S.: Optimal analysis of an online algorithm for the bipartite matching problem on a line. SoCG 2018 pp. 67:1-67:14 (2018) 